\title{
Communication
}

\section{Atomic-scale Structural and Chemical Characterization of Hexagonal Boron Nitride Layers Synthesized at the Wafer-Scale with Monolayer Thickness Control}

\author{
Wei-Hsiang Lin, Victor W. Brar, Deep Jariwala, Michelle C. Sherrott, \\ Wei-Shiuan Tseng, Chih-I Wu, Nai-Chang Yeh, and Harry A Atwater
}

Chem. Mater., Just Accepted Manuscript • Publication Date (Web): 22 May 2017

Downloaded from http://pubs.acs.org on May 23, 2017

\section{Just Accepted}

"Just Accepted" manuscripts have been peer-reviewed and accepted for publication. They are posted online prior to technical editing, formatting for publication and author proofing. The American Chemical Society provides "Just Accepted" as a free service to the research community to expedite the dissemination of scientific material as soon as possible after acceptance. "Just Accepted" manuscripts appear in full in PDF format accompanied by an HTML abstract. "Just Accepted" manuscripts have been fully peer reviewed, but should not be considered the official version of record. They are accessible to all readers and citable by the Digital Object Identifier (DOI®). "Just Accepted" is an optional service offered to authors. Therefore, the "Just Accepted" Web site may not include all articles that will be published in the journal. After a manuscript is technically edited and formatted, it will be removed from the "Just Accepted" Web site and published as an ASAP article. Note that technical editing may introduce minor changes to the manuscript text and/or graphics which could affect content, and all legal disclaimers and ethical guidelines that apply to the journal pertain. ACS cannot be held responsible for errors or consequences arising from the use of information contained in these "Just Accepted" manuscripts. 


\title{
Atomic-scale Structural and Chemical Characterization of Hexagonal Boron Nitride Layers Synthesized at the Wafer-Scale with Monolayer Thickness Control
}

\author{
Wei-Hsiang Lin ${ }^{1}$, Victor W. Brar ${ }^{1,2,3}$, Deep Jariwala ${ }^{1,4}$, Michelle C. Sherrott ${ }^{1,4}$, Wei-Shiuan Tseng ${ }^{5}$, \\ Chih-I Wu ${ }^{6}$, Nai-Chang $\mathrm{Yeh}^{2,5}$, and Harry A. Atwater ${ }^{1,2,4^{*}}$ \\ *haa@caltech.edu \\ 1. Thomas J. Watson Laboratory of Applied Physics, California Institute of Technology, Pasadena, CA 91125, \\ United States \\ 2. Kavli Nanoscience Institute, California Institute of Technology, Pasadena, CA 91125, United States \\ 3. Department of Physics, University of Wisconsin-Madison, Madison, WI 53711, United States \\ 4. Resnick Sustainability Institute, California Institute of Technology, Pasadena, CA 91125, USA. \\ 5. Department of Physics, California Institute of Technology, Pasadena, CA 91125, United States \\ 6. Graduate Institute of Photonics and Optoelectronics and Department of Electrical Engineering, National \\ Taiwan University, Taipei, Taiwan, Republic of China
}

\begin{abstract}
Hexagonal boron nitride (h-BN) is a promising two-dimensional insulator with a large band gap and low density of charged impurities that is isostructural and isoelectronic with graphene. Here we report the chemical and atomic-scale structure of CVD-grown wafer-scale $\left(\sim 25 \mathrm{~cm}^{2}\right)$ h-BN sheets ranging in thickness from 1-20 monolayers. Atomic-scale images of h-BN on $\mathrm{Au}$ and graphene/Au substrates obtained by scanning tunneling microscopy (STM) reveal high h-BN crystalline quality in monolayer samples. Further characterization of 1-20 monolayer samples
\end{abstract}


indicates uniform thickness for wafer-scale areas; this thickness control is a result of precise control of the precursor flow rate, deposition temperature and pressure. Raman and infrared spectroscopy indicate the presence of B-N bonds and reveal a linear dependence of thickness with growth time. X-ray photoelectron spectroscopy (XPS) shows the film stoichiometry, and the $\mathrm{B} / \mathrm{N}$ atom ratio in our films is $1 \pm 0.6 \%$ across the range of thicknesses. Electrical current transport in metal/insulator/metal $(\mathrm{Au} / \mathrm{h}-\mathrm{BN} / \mathrm{Au})$ heterostructures indicates that our CVD-grown h-BN films can act as excellent tunnel barriers with a high hard-breakdown field strength. Our results suggest that large-area h-BN films are structurally, chemically and electronically uniform over the wafer scale, opening the door to pervasive application as a dielectric in layered nanoelectronic and nanophotonic heterostructures. 


\section{Introduction}

Since the isolation of graphene on an insulating substrate ${ }^{1-7}$, a variety of other layered materials have been isolated and characterized, and have opened up an exciting field of research ${ }^{8-11}$. However the electronic quality of two-dimensional (2D) active layers in devices is highly sensitive to their immediate environment owing to the large surface to volume ratio. Therefore a crystalline 2D material that can serve the role of an insulating substrate, encapsulating layer or gate dielectric is highly desirable in device applications. Hexagonal boron nitride (h-BN) has emerged as a promising material for these applications. It has been observed experimentally that encapsulating other 2D materials in h-BN not only enhances the performance of devices but also extends their durability to long time-scales ${ }^{12-13}$. However most previous studies have used mechanically exfoliated h-BN derived from bulk crystals which does not allow for control over layer thickness and sample lateral size. While numerous reports about chemical vapor deposition (CVD) synthesis of h-BN already exist $^{14-32}$ ranging from low pressure CVD (LPCVD) for monolayer growth to ambient pressure CVD (APCVD) for multilayer growth, most of these approaches do not yield precise thickness control from the monolayer scale to thick multilayers over large areas. Many previous experiments have reported on use of solid ammonia-borane as the boron and nitrogen source. The sublimation of a solid source gives poor control of precursor flow rate and partial pressure in the growth chamber over large length scales. Further, monolayer to sub-2nm growth of h-BN is a process catalyzed by the $\mathrm{Cu}$ surface and requires low growth pressures. However the catalytic activity of $\mathrm{Cu}$ surface is diminished after growth of 1-2 $\mathrm{nm}$ h-BN on its surface and thus growth of thicker h-BN films occurs solely via van der Waals epitaxy, requiring higher growth pressures. No system has yet been developed that can achieve both of these growth condition regimes; therefore most prior work on CVD grown h-BN has been able to control both $\mathrm{BN}$ thickness and lateral thickness uniformity over 
technologically relevant areas. Our CVD methods features precursor flow and pressure control systems which combines the merits of both LPCVD and APCVD by allowing precise control over the precursor flow rate and partial pressure of the precursor in the growth zone over a wide range of growth chamber pressure and temperatures. This permits growth of high quality mono to sub 5-layer h-BN films on a $\mathrm{Cu}$ foil which require low growth pressures as well as thicker h-BN films on $\mathrm{Cu}$ foils, by switching to APCVD mode.

We image the atomic structure of monolayer h-BN/Au sheets and monolayer h-BN/graphene/Au heterostructures using scanning tunneling microscopy (STM). Atomic STM images of CVD-grown monolayer h-BN/Au film monolayers and h-BN/graphene heterostructures indicate high crystalline quality. The crystal structure and chemical composition of the resulting h-BN film are also characterized by atomic force microscopy (AFM), Raman spectroscopy, infrared transmission measurements and x-ray photoelectron spectroscopy (XPS). The electrical properties of the thin and thick h-BN films were systematically measured on metal-insulator-metal (MIM) tunneling devices to understand the dielectric properties of these films. Our results demonstrate a new standard and state-of-the-art for large area synthesis of h-BN potentially enabling applications in nanoelectronic and nanophotonic devices.

\section{Results and Discussion}

To grow ultrathin $(<1.5 \mathrm{~nm}) \mathrm{h}-\mathrm{BN}$ films, we use a growth pressure of $\sim 2$ Torr. Figure $1 \mathrm{~b}$ shows an optical photograph and atomic force microscopy (AFM) analysis of a wafer-scale h-BN monolayer which has transferred onto a $285 \mathrm{~nm} \mathrm{SiO}_{2} / \mathrm{Si}$ substrate. Raman spectra acquired over six different spots on the sample (denoted by $\mathrm{x}$ ), as seen in the adjacent plot suggests that the sample thickness and quality is uniform over the entire $\mathrm{cm}^{2}$ scale. Likewise, the growth mode can be 
switched from a slow growth rate of $\sim 0.3 \mathrm{~nm} / \mathrm{min}$ in catalytically controlled CVD at low pressures to

a high growth rate of $1 \mathrm{~nm} / \mathrm{min}$ at near atmospheric pressures ( $\sim 500$ Torr). Figure 1c illustrates the

large area uniformity of $\sim 15$ monolayer h-BN as inferred from the optical micrograph and

corresponding Raman spectra. To validate the precise control of layer thickness and growth

uniformity, a series of growth experiments were performed for varying times at both low and

ambient pressures to produce h-BN films of varying layer number/thicknesses. These h-BN films

were then characterized with several spectroscopic techniques to characterize their structure and

chemical composition. Atomic scale structure and areal homogeneity of h-BN films were revealed

by scanning tunneling microscopy (STM) for CVD-grown monolayer h-BN. Despite numerous

reports on CVD synthesis of h-BN, little is known about atomic scale electronic structure for layers

grown on polycrystalline $\mathrm{Cu}$ foils, due to the roughness of the $\mathrm{Cu}$ foil substrate which renders STM

difficult. Also, the film thickness inhomogeneity seen in most prior reports ${ }^{33-37}$ would prevent image

formation by direct electron tunneling through h-BN. Notably, h-BN is difficult to characterize using

STM due to its insulating character. To enable adequate sample conductance, many research groups

have used a graphene/ h-BN heterostructure which exploits the conductance of graphene to visualize

the atomic structure of the h-BN layer underneath the graphene. In contrast, we are able to use STM

to directly image our CVD-grown monolayer h-BN films on $\mathrm{Au}$ (111)/mica substrates transferred 
using polymer free transfer method ${ }^{38}$. Figure $2 \mathrm{a}$ is a schematic of the STM measurement

configuration for monolayer h-BN sheets on $\mathrm{Au}$ (111) substrates. Figure $2 \mathrm{~b}$ shows a representative

STM image of the monolayer h-BN sheet on $\mathrm{Au}(111)$ without any post transfer annealing. The

atomically-resolved h-BN honeycomb structure is clearly visible, superimposed on the $\mathrm{Au}(111)$

herringbone reconstruction pattern. The appearance of the distorted hexagonal lattice in the STM

image indicates strong surface tension originating from the interaction between boron nitride atoms

and the $\mathrm{Au}$ (111) substrate herringbone reconstruction. Figure 2c shows schematic of STM

measurements of the monolayer h-BN sheet on monolayer graphene/Au (111) substrate and Figure

2d, e show topographic STM images acquired from two different areas of the single-layer h-BN on

graphene. Both the atomic lattices of h-BN and longer range moiré patterns can be clearly revealed.

The moiré pattern is formed by interference between the h-BN layer and underlying graphene/Au

(111) substrate, and can be attributed to their lattice mismatch $(a=0.252 \mathrm{~nm}, b=0.246 \mathrm{~nm})$ and

rotational misalignment. The h-BN and graphene sheets interact through van der Waals forces, and

display the same topographic conformal mapping to the underlying Au (111). However, the relative

rotation angle between the graphene and h-BN sheets can be modified by tiny wrinkles and bubbles

are inevitably introduced during the transfer process of h-BN onto graphene as seen in Fig. 2d (left)

and original grain boundary of $\mathrm{h}-\mathrm{BN}$ and graphene. Changing the rotation angles between the 
h-BN and graphene lattices, leads to moiré patterns with different periodicities and orientations as

observed in Figure 2d and e (center). The periodicity of moiré pattern presented in Figure 2d is 3.4

$\mathrm{nm}$ and $2.0 \mathrm{~nm}$ in Figure 2e. The twist angle between the h-BN and graphene lattices can be

ascertained from the structure of the Moire pattern and is given by

$$
\theta=\cos ^{-1}\left[1-\frac{a^{2} b^{2}-\lambda^{2}(b-a)^{2}}{2 a b \lambda^{2}}\right]
$$

where $\mathrm{a}$ is the h-BN lattice constant, $\mathrm{b}$ is the graphene lattice constant, and $\lambda$ is the periodicity of the moiré pattern. Thus, the twist angle $\theta$ of Figure $2 \mathrm{~d}$ and $2 \mathrm{e}$ is found to be $(4 \pm 0.1)^{\circ}$ and $(7 \pm$ $0.1)^{\circ}$, respectly. Alternatively, the twist angle $\theta$ can also be extracted by performing a fast Fourier transform (FFT) analysis of the STM images, as shown in the inset of Figure $2 \mathrm{~d}$ and 2e. The outside set of spots corresponds to the reciprocal lattice of h-BN, while the inner set of spots are assigned to the moiré pattern stemming from the rotation between the monolayer h-BN and graphene substrate. The atomic resolution STM images presented here can be achieved in image locations over a wide area of the wafer-scale sample, indicating excellent h-BN sheet surface quality for layers transferred with the polymer-free ${ }^{38}$ transfer method and supporting the existence of high crystalline quality in CVD-grown h-BN down to the monolayer level. The STM results provide information about the atomic scale structure and crystallinity of monolayer h-BN. However they do not provide information about macroscopic-scale h-BN film thickness homogeneity, composition or structure. 
Raman spectra of an h-BN layer typically have two active $E_{2 g}$ modes, one at $1366 \mathrm{~cm}^{-1}$ which is strong and corresponds to vibrations of $\mathrm{B}$ and $\mathrm{N}$ moving against each other in the plane and another at $51.8 \mathrm{~cm}^{-1}$, which is attributed to sliding between whole planes. However the lower frequency mode is more difficult to observe because of proximity to the Rayleigh diffusion, as well as the presence of a fluorescence background. The width, intensity and position of these Raman features are sensitive to h-BN thickness, and these dependencies were determined by combining Raman measurement with STM results and AFM results. We use monolayer h-BN as a thickness calibration for every run and verify the integrated intensity of this calibration point with the value marked with an arrow in Figure $3 b$ (Top). The integrated intensity of the h-BN layers determined using this protocol gives a reasonable estimate of the layer thickness and matches very well with the estimates given by the AFM line profiles (see supporting information S1). Figure 3a shows Raman spectra of monolayer h-BN to 15 layers h-BN films, showing the Raman intensity increases with the number of layers. The integrated intensity is plotted as a function of the number of layers in Figure $3 b$ (Top). Figure $3 \mathrm{~b}$ (Bottom) suggests that the peak position have a redshift as layers decrease and the FWHM become shaper as the layer number decreases. While Raman spectroscopy suggests the presence of B-N bonds and a linear dependence of thickness with growth time, they do not provide any information on the stoichiometry of the grown films. To probe the chemical composition, we used 
$\mathrm{X}$-ray photoelectron spectroscopy (XPS) to determine the $\mathrm{B} / \mathrm{N}$ ratio. Figure $3 \mathrm{c}$ shows the XPS

spectra of as-grown $\mathrm{h}-\mathrm{BN}$ films on a $\mathrm{Cu}$ foils with a film thickness varying from monolayer to 30

layers. It has been previously reported ${ }^{39}$ that bulk boron nitride with hexagonal phase exhibits a B 1s

core level at $190.1 \mathrm{eV}$. Figure 3c (left) shows XPS B1s core level spectra with a peak center at 190.2

$\mathrm{eV}$, which is very close to the h-BN bulk phase value. Figure $3 \mathrm{c}$ (right) shows that the $\mathrm{N} 1 \mathrm{~s}$ peak is

located at $397.7 \mathrm{eV}$, similar to the reported position of the N1s spectrum $(398.1 \mathrm{eV})$ for h-BN. Both

the $\mathrm{B} 1 \mathrm{~s}$ and $\mathrm{N}$ 1s spectra indicate that the configuration for $\mathrm{B}$ and $\mathrm{N}$ atoms is the $\mathrm{B}-\mathrm{N}$ bond, implying that the hexagonal phase is the phase of our BN films. Further, it can also be seen that the intensities of the B1s and N1s increases with increasing layer thickness of our films. In addition, we also observe that the intensity of the $\mathrm{Cu} 2 \mathrm{p}$ peak weakens with the increasing thickness of the h-BN films (see supporting information S3) further corroborating our precise thickness controlled growth. Finally, quantitative analysis of the $\mathrm{B} 1 \mathrm{~s}$ and $\mathrm{N} 1 \mathrm{~s}$ spectra indicates that the $\mathrm{B} / \mathrm{N}$ atom ratio in our films was $1 \pm 0.6 \%$ across the range of thicknesses. These results evidently confirm growth of high quality h-BN layer and continuous film on $\mathrm{Cu}$ foil using ammonia borane by our optimized CVD process. The large area uniformity and high crystalline quality of our CVD grown h-BN films makes them ideal for applications as ultrathin dielectrics in optoelectronic devices ${ }^{38,40-41}$. Therefore, to evaluate the dielectric strength and leakage through our h-BN films, we investigate the electronic 
properties of tunnel junctions in which h-BN acts as a barrier layer between two gold electrodes. The dielectric properties of the CVD h-BN films with different thicknesses were measured by fabricating metal/h-BN/metal (MIM) capacitors and measuring current-voltage (I-V) characteristics. Figure 4a shows a schematic diagram of an $\mathrm{Au} / \mathrm{h}-\mathrm{BN} / \mathrm{Au}$ capacitor. We use template stripped gold films as our bottom electrode with root mean square (RMS) roughness less than $0.5 \mathrm{~nm}$. The h-BN films of various thicknesses were transferred onto the template stripped gold. Then, we used standard electron beam deposition techniques to deposit 100nm gold through a shadow mask to define the top electrodes. The contact area was $10 \mu \mathrm{m} \times 10 \mu \mathrm{m}$. Figure $4 \mathrm{~b}$ shows $\mathrm{I}-\mathrm{V}$ measurements of $\mathrm{h}$-BN layers with various thicknesses from 1 to 15 layers. Mono-, bi-, and 4-layer samples show measurable low-bias conductance, which we ascribe to direct tunneling. Thicker samples are insulating at low bias and show sharp increases at a breakdown voltage that increases with thickness. The inset in the Figure $4 \mathrm{~b}$ shows the conductance as a function of sample thickness, which decays exponentially, as expected for direct tunneling. The current densities at the two metal electrodes and through the h-BN layers of different thicknesses were investigated as a function of voltage, as plotted in Figure 4c. As shown in Figure 4c, the measured currents of the thin h-BN films agreed well with the Poole-Frenkel (PF) emission model, indicating that a trap-assisted PF emission mechanism dominated the transport mechanism for the leakage current in our h-BN films. The Figure 4c shows the PF plot using the 
following equation:

$$
I(V)_{P F}=A q N_{c} \mu V \operatorname{dexp}\left[\frac{-q\left(\Phi_{T}-\sqrt{\frac{q V d}{\pi \varepsilon_{0} \varepsilon_{r}}}\right)}{k T}\right]
$$

where $\mathrm{A}, \mathrm{q}, \mathrm{N}_{\mathrm{c}}, \mu, \Phi_{\mathrm{T}}, \mathrm{V}, \mathrm{d}$ and $\mathrm{h}$ are the effective area, electron charge, density of state in the conduction band, electronic mobility in the oxide, trap energy level in the h-BN, voltage, h-BN thickness and Planck's constant respectively. Finally, for thicker ( $>1 \mathrm{~nm}) \mathrm{h}-\mathrm{BN}$ films we performed irreversible dielectric breakdown measurements to determine the hard-breakdown voltage (see supporting information S 4) and corresponding field strength of the ultrathin h-BN. Figure 4 (d) plots the breakdown field strength as a function of the h-BN thickness. In h-BN films with a thicknesses less than $5 \mathrm{~nm}$, the breakdown voltage increased linearly with h-BN thickness, indicating very high quality films at the few-layer limit. Breakdown field strength approaching $\sim 4.3 \mathrm{MV} / \mathrm{cm}$ were observed for $4.5 \mathrm{~nm}$ thick BN films.

\section{Conclusions}

We have imaged the atomic-scale structure of monolayer h-BN sheets on $\mathrm{Au}$, and moiré patterns on monolayer h-BN/graphene heterostructures using scanning tunneling microscopy (STM). Atomic STM images of monolayer h-BN film and moiré patterns on monolayer h-BN/graphene heterostructures show the high crystalline quality of the CVD grown h-BN up to the atomic level. 


\begin{abstract}
We also introduced a hybrid LP and APCVD system that uses controlled precursor to grow uniform, layer by layer thickness controlled wafer scale h-BN films with thicknesses ranging from monolayer to $10 \mathrm{~nm}$. Spectroscopic characterization suggests that the films are stoichiometric and highly uniform over wafer-scale areas. Electrical measurements for metal-insulator-metal ( $\mathrm{Au} / \mathrm{h}-\mathrm{BN} / \mathrm{Au})$ structures indicate that our CVD-grown h-BN films can act as an excellent tunnel barrier with a high hard-breakdown field strength. Successful large area CVD growth of h-BN films defines a new state-of-the-art for application of this material in future large-area, electronic and photonic devices.
\end{abstract}


Methods

Pre-treatment of copper foil. Copper foil (25 $\mu \mathrm{m}, 99.999 \%$ pure, Alfa Aesar, item no. 10950) was soaked and sonicated in acetone and isopropyl alcohol (IPA) for 30 min consecutively to remove organic impurities. Then, it was washed with deionized water and dried with nitrogen gas.

Synthesis of mono- and multilayer h-BN. The h-BN films were grown using a home-built hybrid CVD setup. Figure 1a shows the schematic of the growth setup. The setup comprises of a $52 \mathrm{~mm}$ inner diameter (I.D.) horizontal split tube furnace (MTI Corporation). The solid precursor ammonia-borane $\left(\mathrm{NH}_{3}-\mathrm{BH}_{3}\right)$ powder, (97\% purity, Sigma-Aldrich) is contained in a home-made quartz container, attached to the main growth chamber (22 mm I.D. quartz tube) via a leak valve and heated separately from the quartz tube via use of a resistive heating belt. $\mathrm{Cu}$ foils $(25 \mu \mathrm{m}, 99.999 \%$ pure, Alfa Aesar) are used as the catalytic growth substrates. The pressure in the growth chamber can be independently controlled via an angle valve at the vacuum pump while the pressure in the precursor bubbler can be controlled via the leak valve and carrier gas flow rates. The monolayer and multilayer h-BN was synthesized using a pressure controllable CVD system. The copper foil was inserted into the center of a $22 \mathrm{~mm}$ I.D. quartz tube, heated by a horizontal split-tube furnace. The Ammonia borane $\left(\mathrm{NH}_{3}-\mathrm{BH}_{3}\right)$ (97\% purity, from Sigma-Aldrich), stable in an atmospheric environment, was used as the precursor. It was loaded in a homemade quartz container which is 
isolated from the main CVD system with a leak valve to control the flow rate. The quartz tube inlet and outlet were blocked by the filters to prevent the BN nanoparticles from diffusing into the gas line. First, the quartz tube was pump down to $5 \times 10^{-3}$ torr, and then ultrahigh purity grade hydrogen gas was introduced during the temperature ramp-up of the furnace (pressure $\sim 100$ mtorr, flow rate $\sim 50 \mathrm{sccm}$ ). The copper foil was annealed at $950^{\circ} \mathrm{C}$ in hydrogen for $60 \mathrm{~min}$ to obtain a smooth surface. After annealing, the ultrahigh purity argon gas $(300 \mathrm{sccm})$ was introduced into the system and waited for $30 \mathrm{~min}$ to stable the tube environment. The precursor was heated to $130^{\circ} \mathrm{C}$ and decomposed to hydrogen gas, monomeric aminoborane, and borazine gas. After the precursor temperature reach $130^{\circ} \mathrm{C}$, the manual valve between quartz tube outlet and the pump was slowly closed and stopped until the pressure reach 20 torr. When the desired pressure was achieved, the leak valve to the precursor was open. The typical growth time is $3 \mathrm{~min}$ for monolayer h-BN layer and 20 min for the $20 \mathrm{~nm}$ thickness h-BN layer. To atomically control the thickness of h-BN layer, it is very important to have a leak valve to control the flow rate of the precursor. Also, we can change the growth rate of the h-BN by changing the pressure of the growth environment. After growth, the tube furnace was cooled down with the cooling rate $\sim 16^{\circ} \mathrm{C} / \mathrm{min}$.

Transfer of mono- and multilayer h-BN. To transfer h-BN onto a target substrate, the conventional poly(methyl methacrylate) (PMMA) transfer method and polymer-free transfer method ${ }^{41}$ were 
applied based on different purposes.

Characterization. Atomic Force Microscopy (AFM) (Bruker Dimension Icon), were done using tapping mode to characterize the surface morphology of the h-BN film transferred on the $\mathrm{SiO}_{2} / \mathrm{Si}$ substrate. The quality of the h-BN film was characterized using the Raman spectroscopy, X-ray photoemission spectroscopy (XPS) and Scanning tunneling microscopy. The Raman spectra were taken with a Renishaw M1000 micro-Raman spectrometer system using a $514.3 \mathrm{~nm}$ laser $(2.41 \mathrm{eV})$ as the excitation source. A $50 \mathrm{X}$ objective lens with a numerical aperture of 0.75 and a 2400 lines/mm grating were chosen during the measurement to achieve better signal-to-noise ratio.

XPS was performed under $10^{-9}$ torr with a Surface Science Instruments M-Probe instrument utilizing $\mathrm{Al} \mathrm{K}_{\alpha} \mathrm{X}$-rays and a hemispherical energy analyzer. STM was carried out with an Omicron system at room temperature.

\section{Acknowledgements}

The authors gratefully acknowledge support from the Department of Energy, Office of Science under Grant DE-FG02-07ER46405 (W.H.L. and H.A.A.) and for use of facilities of the DOE “ Light-Material Interactions in Energy Conversion ” Energy Frontier Research Center (DE-SC0001293). D.J. and M.C.S. acknowledge additional support from Resnick Sustainability 
Institute Graduate and Postdoctoral Fellowships whereas V.W.B. acknowledge additional support from the Kavli Nanoscience Postdoctoral Fellowship. The authors thank Prof. George Rossman for access to the Raman and FTIR tools. The authors thank I-Te Lu for the discussion about the moiré pattern calculation. The authors acknowledge support from the Beckman Institute of the California Institute of Technology to the Molecular Materials Research Center.

\section{Competing financial interests}

The authors declare no competing financial interests. 
Figure 1.

(a)

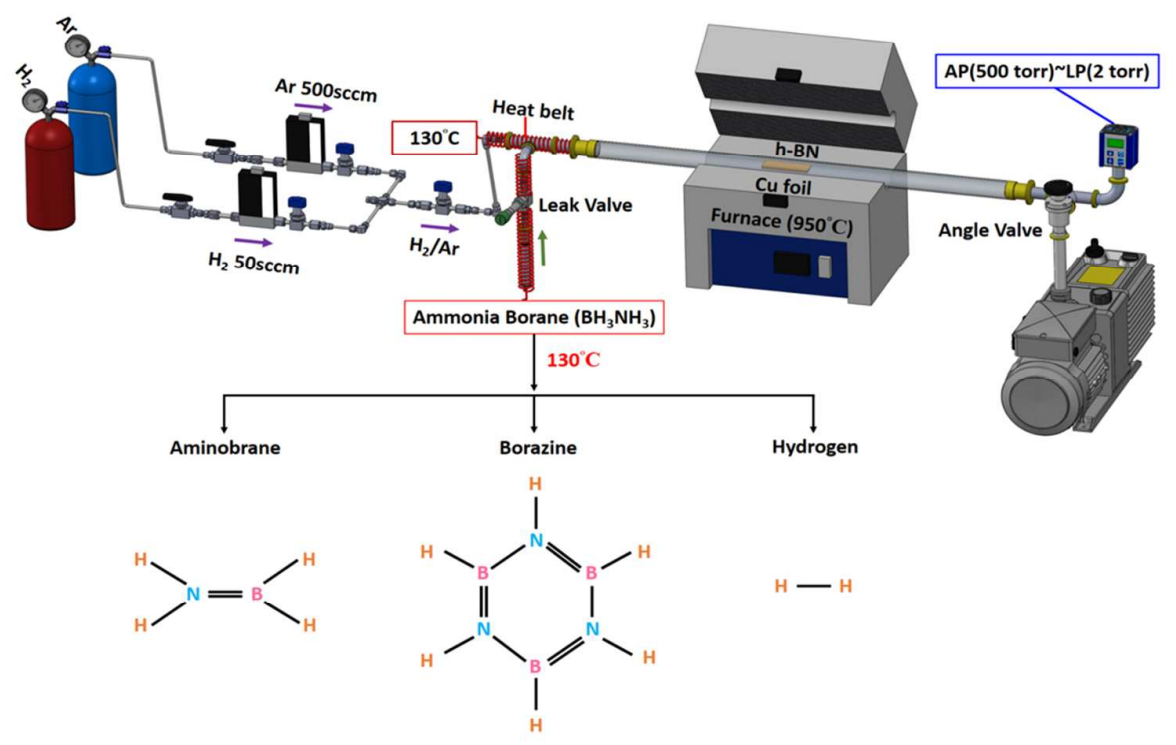

(b)

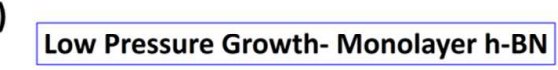

(c)

Atmospheric Pressure Growth- Multilayer h-BN
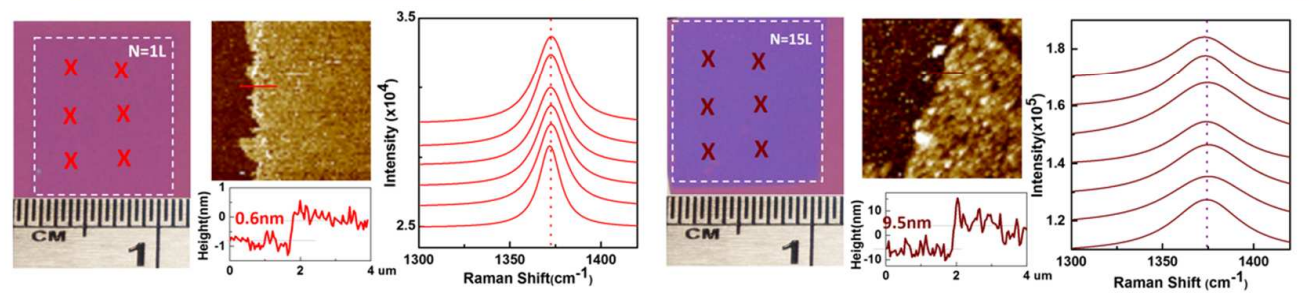

Figure 1. (a) Schematic diagram of hybrid atmospheric pressure and low pressure CVD system used for h-BN grow. (b) Photograph of a large and uniform monolayer h-BN film on a $285 \mathrm{~nm}$ thick $\mathrm{SiO}_{2} / \mathrm{Si}$ substrate, and corresponding AFM image and Raman spectra. (c) Photograph of a large and uniform multilayer h-BN film on a $285 \mathrm{~nm}$ thick $\mathrm{SiO}_{2} / \mathrm{Si}$ substrate, and corresponding AFM image and Raman spectra. 
Figure 2.
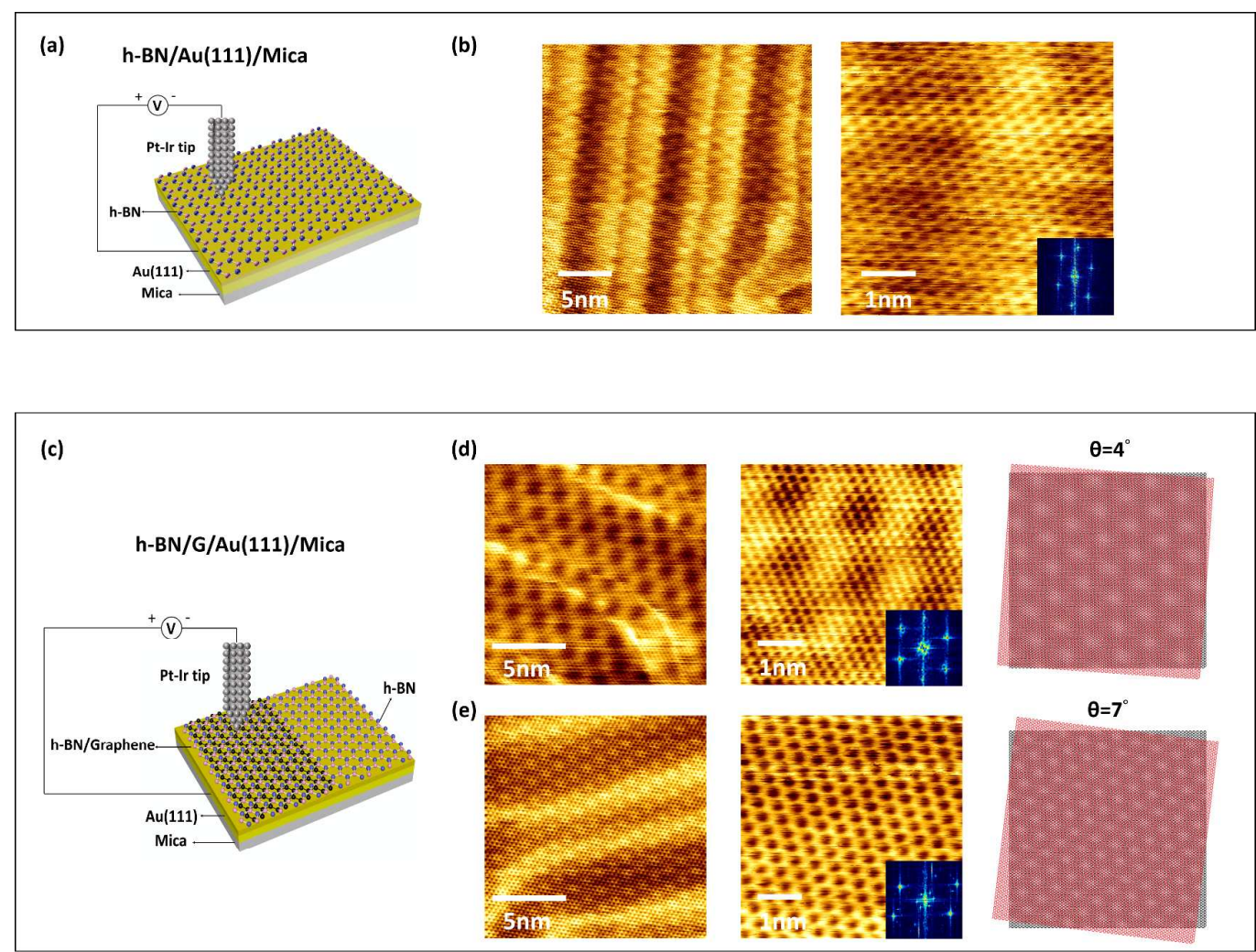

Figure 2. (a) STM measurement schematics on monolayer h-BN film. (b) The representative STM image of the h-BN after transfer to the $\mathrm{Au}\left(\begin{array}{lll}1 & 1 & 1\end{array}\right)$ substrate, with $\mathrm{V}_{\text {sample }}=0.5 \mathrm{~V}$ and $\mathrm{I}_{\text {tunnel }}=0.5 \mathrm{nA}$. The image size is $25 \mathrm{~nm} \times 25 \mathrm{~nm}$ (Left) and $5 \mathrm{~nm} \times 5 \mathrm{~nm}$ (Right). (c) STM measurement schematics on monolayer h-BN/graphene heterostructure. (d) Topography of a moiré superlattice with periodicity of $3.7 \mathrm{~nm}$ and a $4^{\circ}$ twist between h-BN and graphene. The image was acquired under $\mathrm{V}_{\text {sample }}=0.5 \mathrm{~V}_{\text {and }} \mathrm{I}_{\text {tunnel }}=0.5 \mathrm{nA}$ and image size is $15 \mathrm{~nm} \times 15 \mathrm{~nm}$ (Left) and $5 \mathrm{~nm} \times 5 \mathrm{~nm}$ (Right). (e) Topography of a moiré superlattice with periodicity of $2.0 \mathrm{~nm}$ and a $7^{\circ}$ twist between $\mathrm{h}-\mathrm{BN}$ and graphene. The image was acquired under $\mathrm{V}_{\text {sample }}=0.5 \mathrm{~V}$ and $\mathrm{I}_{\text {tunnel }}=0.5 \mathrm{nA}$ and image size is $15 \mathrm{~nm}$ $\times 15 \mathrm{~nm}$ (Left) and $5 \mathrm{~nm} \times 5 \mathrm{~nm}$ (Right). The insets of (d) and (e) are Fourier transform patterns of corresponding STM images. 
Figure 3.

(a)

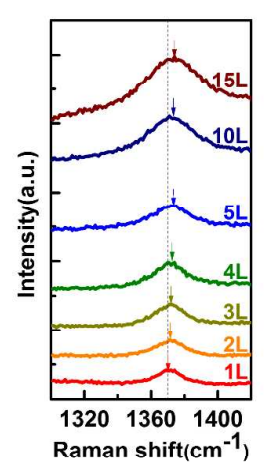

(b)

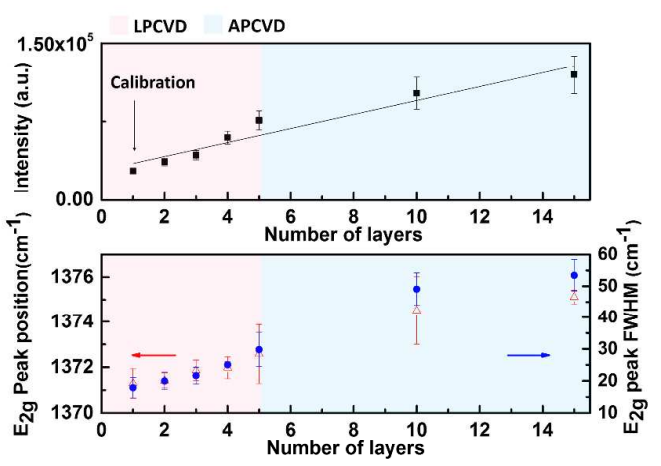

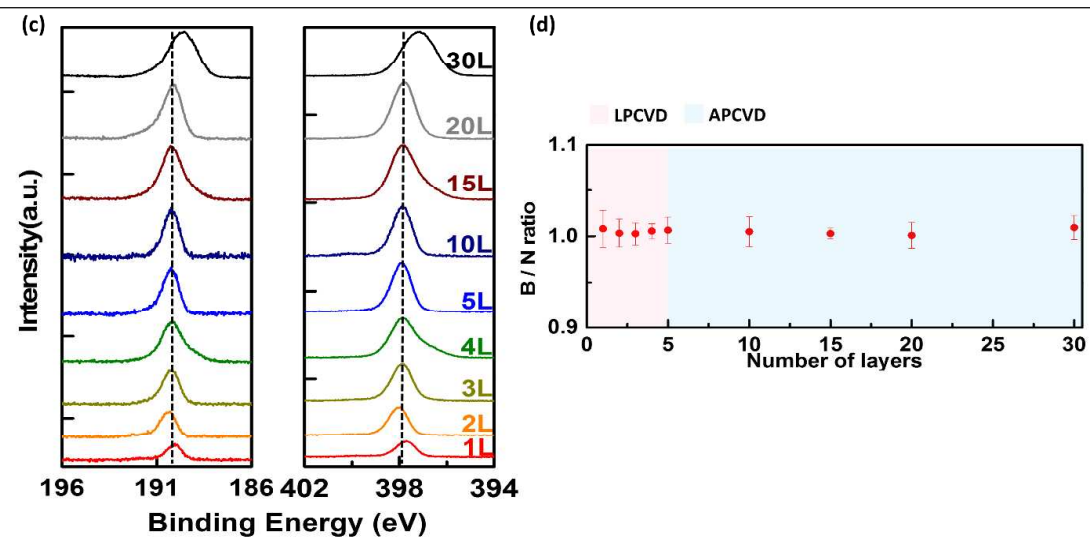

Figure 3. (a) Raman spectra for h-BN layers with 1-15 atomic layers. (b) (Top) Integrated intensity shows a steady increase with increase in the layer number of h-BN. (b) (Bottom left) The position of $\mathrm{E}_{2 \mathrm{~g}}$ peak vs. number of monolayers of $\mathrm{h}-\mathrm{BN}$, showing the blue shift with increased number of $\mathrm{h}-\mathrm{BN}$ layers. (Bottom right) The Full width at half maximum vs. number of h-BN layers, showing a steady increase of the FWHM with h-BN thickness. (c) X-ray photoelectron spectra (XPS) from different thickness of h-BN layers on $\mathrm{Cu}$ foil. (d) High resolution B1s and N1s peaks corresponding to the thickness from $1 \sim 30$ layers h-BN films on Cu foil. (Right) Quantitative analysis of the B1s and N1s spectra indicates that the $\mathrm{B} / \mathrm{N}$ atom ratio in our films was $1 \pm 0.6 \%$ across the range of thicknesses. 
Figure 4.

(a)

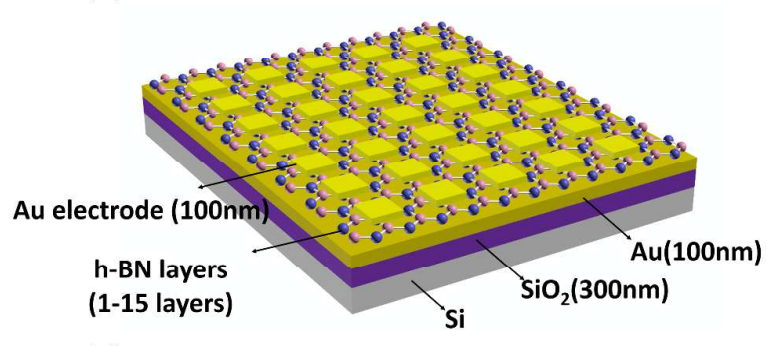

(c)

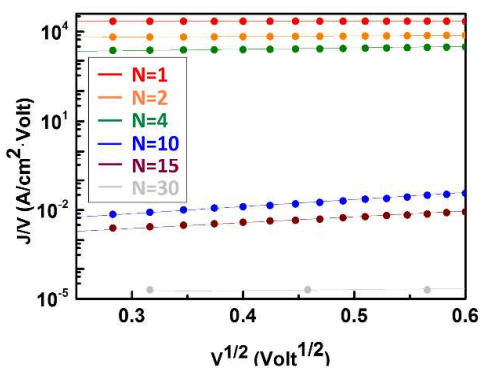

(b)

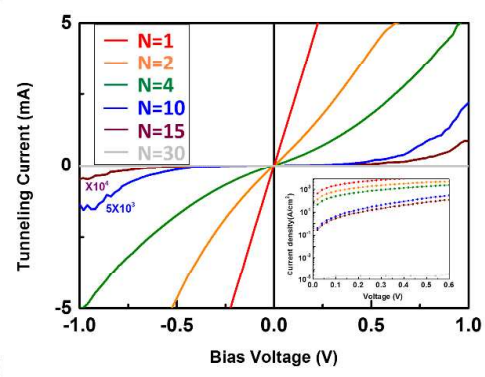

(d)

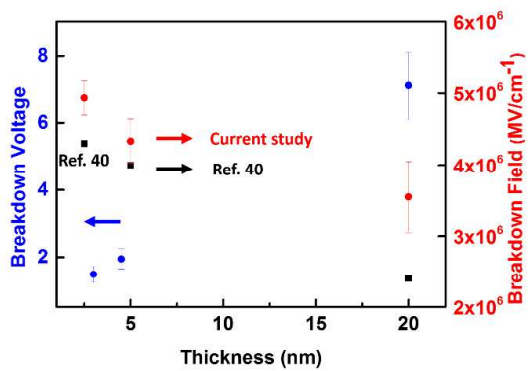

Figure 4. (a) A schematic diagram of the $\mathrm{Au} / \mathrm{h}-\mathrm{BN} / \mathrm{Au}(\mathrm{MIM})$ capacitors fabricated on a Si substrate. (b) Characteristic I-V curves for Au / h-BN / Au devices with different thicknesses of BN insulating layer: red curve, monolayer of $\mathrm{BN}$; orange, bilayer; green, four layer; navy, 10 layers; and purple, 15 layers. The inset of (a) is typical J-V characteristics of a MIM capacitor, described by the field-assisted tunneling model. The h-BN thickness range was less than $5 \mathrm{~nm}$. The inset shows a PF emission plot $\left(\mathrm{J} / \mathrm{V}\right.$ versus $\left.1 / \mathrm{V}^{1 / 2}\right)$. (d) The breakdown characteristics as a function of the h-BN film thickness. 


\section{Reference}

1. Dean, C. R.; Young, A. F.; Meric, I.; Lee, C.; Wang, L.; Sorgenfrei, S.; Watanabe, K.; Taniguchi, T.; Kim, P.; Shepard, K. L.; Hone, J., Boron nitride substrates for high-quality graphene electronics. Nat Nanotechnol 2010, 5, 722-726.

2. Kim, K. K.; Hsu, A.; Jia, X. T.; Kim, S. M.; Shi, Y. M.; Dresselhaus, M.; Palacios, T.; Kong, J., Synthesis and Characterization of Hexagonal Boron Nitride Film as a Dielectric Layer for Graphene Devices. Acs Nano 2012, 6, 8583-8590.

3. Zhang, K.; Yap, F. L.; Li, K.; Ng, C. T.; Li, L. J.; Loh, K. P., Large Scale Graphene/Hexagonal Boron Nitride Heterostructure for Tunable Plasmonics. $A d v$ Funct Mater 2014, 24, 731-738.

4. Novoselov, K. S.; Geim, A. K.; Morozov, S. V.; Jiang, D.; Katsnelson, M. I.; Grigorieva, I. V.; Dubonos, S. V.; Firsov, A. A., Two-dimensional gas of massless Dirac fermions in graphene. Nature 2005, 438, 197-200.

5. Levendorf, M. P.; Kim, C. J.; Brown, L.; Huang, P. Y.; Havener, R. W.; Muller, D. A.; Park, J., Graphene and boron nitride lateral heterostructures for atomically thin circuitry. Nature 2012, 488, 627-632.

6. Kim, S. M.; Hsu, A.; Park, M. H.; Chae, S. H.; Yun, S. J.; Lee, J. S.; Cho, D. H.; Fang, W. J.; Lee, C.; Palacios, T.; Dresselhaus, M.; Kim, K. K.; Lee, Y. H.; Kong, J., Synthesis of large-area multilayer hexagonal boron nitride for high material performance. Nat Commun 2015, 6, 8662

7. Geim, A. K.; Novoselov, K. S., The rise of graphene. Nat Mater 2007, 6 , 183-191.

8. Radisavljevic, B.; Radenovic, A.; Brivio, J.; Giacometti, V.; Kis, A., Single-layer MoS2 transistors. Nat Nanotechnol 2011, 6, 147-150.

9. Fu, L.; Sun, Y. Y.; Wu, N.; Mendes, R. G.; Chen, L. F.; Xu, Z.; Zhang, T.; Rummeli, M. H.; Rellinghaus, B.; Pohl, D.; Zhuang, L.; Fu, L., Direct Growth of MoS2/h-BN Heterostructures via a Sulfide-Resistant Alloy. Acs Nano 2016, 10, 2063-2070.

10. Doganov, R. A.; Koenig, S. P.; Yeo, Y. T.; Watanabe, K.; Taniguchi, T.; Ozyilmaz, B., Transport properties of ultrathin black phosphorus on hexagonal boron nitride. Appl Phys Lett 2015, 106, 083505.

11. Kim, C. J.; Brown, L.; Graham, M. W.; Hovden, R.; Havener, R. W.; McEuen, P. L.; Muller, D. A.; Park, J., Stacking Order Dependent Second Harmonic Generation and Topological Defects in h-BN Bilayers. Nano Lett 2013, 13, 5660-5665.

12. Ci, L.; Song, L.; Jin, C. H.; Jariwala, D.; Wu, D. X.; Li, Y. J.; Srivastava, A.; 
Wang, Z. F.; Storr, K.; Balicas, L.; Liu, F.; Ajayan, P. M., Atomic layers of hybridized boron nitride and graphene domains. Nat Mater 2010, 9, 430-435.

13. Zhu, J.; Kang, J.; Kang, J. M.; Jariwala, D.; Wood, J. D.; Seo, J. W. T.; Chen, K. S.; Marks, T. J.; Hersam, M. C., Solution-Processed Dielectrics Based on Thickness-Sorted Two-Dimensional Hexagonal Boron Nitride Nanosheets. Nano Lett 2015, 15, 7029-7036.

14. Song, L.; Ci, L. J.; Lu, H.; Sorokin, P. B.; Jin, C. H.; Ni, J.; Kvashnin, A. G.; Kvashnin, D. G.; Lou, J.; Yakobson, B. I.; Ajayan, P. M., Large Scale Growth and Characterization of Atomic Hexagonal Boron Nitride Layers. Nano Lett 2010, 10 , 3209-3215.

15. Shi, Y. M.; Hamsen, C.; Jia, X. T.; Kim, K. K.; Reina, A.; Hofmann, M.; Hsu, A. L.; Zhang, K.; Li, H. N.; Juang, Z. Y.; Dresselhaus, M. S.; Li, L. J.; Kong, J., Synthesis of Few-Layer Hexagonal Boron Nitride Thin Film by Chemical Vapor Deposition. Nano Lett 2010, 10, 4134-4139.

16. Liu, Z.; Song, L.; Zhao, S. Z.; Huang, J. Q.; Ma, L. L.; Zhang, J. N.; Lou, J.; Ajayan, P. M., Direct Growth of Graphene/Hexagonal Boron Nitride Stacked Layers. Nano Lett 2011, 11, 2032-2037.

17. Gorbachev, R. V.; Riaz, I.; Nair, R. R.; Jalil, R.; Britnell, L.; Belle, B. D.; Hill, E. W.; Novoselov, K. S.; Watanabe, K.; Taniguchi, T.; Geim, A. K.; Blake, P., Hunting for Monolayer Boron Nitride: Optical and Raman Signatures. Small 2011, 7, 465-468. 18. Britnell, L.; Gorbachev, R. V.; Jalil, R.; Belle, B. D.; Schedin, F.; Katsnelson, M. I.; Eaves, L.; Morozov, S. V.; Mayorov, A. S.; Peres, N. M. R.; Neto, A. H. C.; Leist, J.; Geim, A. K.; Ponomarenko, L. A.; Novoselov, K. S., Electron Tunneling through Ultrathin Boron Nitride Crystalline Barriers. Nano Lett 2012, 12, 1707-1710.

19. Lee, K. H.; Shin, H. J.; Lee, J.; Lee, I. Y.; Kim, G. H.; Choi, J. Y.; Kim, S. W., Large-Scale Synthesis of High-Quality Hexagonal Boron Nitride Nanosheets for Large-Area Graphene Electronics. Nano Lett 2012, 12, 714-718.

20. Guo, N.; Wei, J. Q.; Fan, L. L.; Jia, Y.; Liang, D. Y.; Zhu, H. W.; Wang, K. L.; $\mathrm{Wu}, \mathrm{D}$. H., Controllable growth of triangular hexagonal boron nitride domains on copper foils by an improved low-pressure chemical vapor deposition method. Nanotechnology 2012, 23, 415605.

21. Ismach, A.; Chou, H.; Ferrer, D. A.; Wu, Y. P.; McDonnell, S.; Floresca, H. C.; Covacevich, A.; Pope, C.; Piner, R.; Kim, M. J.; Wallace, R. M.; Colombo, L.; Ruoff, R. S., Toward the Controlled Synthesis of Hexagonal Boron Nitride Films. Acs Nano 2012, 6, 6378-6385. 
22. Wang, M.; Jang, S. K.; Jang, W. J.; Kim, M.; Park, S. Y.; Kim, S. W.; Kahng, S. J.; Choi, J. Y.; Ruoff, R. S.; Song, Y. J.; Lee, S., A Platform for Large-Scale Graphene Electronics - CVD Growth of Single-Layer Graphene on CVD-Grown Hexagonal Boron Nitride. Adv Mater 2013, 25, 2746-2752.

23. Gibb, A. L.; Alem, N.; Chen, J. H.; Erickson, K. J.; Ciston, J.; Gautam, A.; Linck, M.; Zettl, A., Atomic Resolution Imaging of Grain Boundary Defects in Monolayer Chemical Vapor Deposition-Grown Hexagonal Boron Nitride. J Am Chem Soc 2013, $135,6758-6761$.

24. Kim, G.; Jang, A. R.; Jeong, H. Y.; Lee, Z.; Kang, D. J.; Shin, H. S., Growth of High-Crystalline, Single-Layer Hexagonal Boron Nitride on Recyclable Platinum Foil. Nano Lett 2013, 13, 1834-1839.

25. Liu, Z.; Gong, Y. J.; Zhou, W.; Ma, L. L.; Yu, J. J.; Idrobo, J. C.; Jung, J.; MacDonald, A. H.; Vajtai, R.; Lou, J.; Ajayan, P. M., Ultrathin high-temperature oxidation-resistant coatings of hexagonal boron nitride. Nat Commun 2013, 4, 2541.

26. Tay, R. Y.; Griep, M. H.; Mallick, G.; Tsang, S. H.; Singh, R. S.; Tumlin, T.; Teo, E. H. T.; Karna, S. P., Growth of Large Single-Crystalline Two-Dimensional Boron Nitride Hexagons on Electropolished Copper. Nano Lett 2014, 14, 839-846.

27. Park, J. H.; Park, J. C.; Yun, S. J.; Kim, H.; Luong, D. H.; Kim, S. M.; Choi, S. H.; Yang, W.; Kong, J.; Kim, K. K.; Lee, Y. H., Large-Area Monolayer Hexagonal Boron Nitride on Pt Foil. Acs Nano 2014, 8, 8520-8528.

28. Feigelson, B. N.; Bermudez, V. M.; Hite, J. K.; Robinson, Z. R.; Wheeler, V. D.; Sridhara, K.; Hernandez, S. C., Growth and spectroscopic characterization of monolayer and few-layer hexagonal boron nitride on metal substrates. Nanoscale 2015, 7 , 3694-3702.

29. Wu, Q.; Jang, S. K.; Park, S.; Jung, S. J.; Suh, H.; Lee, Y. H.; Lee, S.; Song, Y. J., In situ synthesis of a large area boron nitride/graphene monolayer/boron nitride film by chemical vapor deposition. Nanoscale 2015, 7, 7574-7579.

30. Stehle, Y.; Meyer, H. M.; Unocic, R. R.; Kidder, M.; Polizos, G.; Datskos, P. G.; Jackson, R.; Smirnov, S. N.; Vlassiouk, I. V., Synthesis of Hexagonal Boron Nitride Mono layer: Control of Nucleation and Crystal Morphology. Chem Mater 2015, 27, 8041-8047.

31. Khan, M. H.; Huang, Z. G.; Xiao, F.; Casillas, G.; Chen, Z. X.; Molino, P. J.; Liu, H. K., Synthesis of Large and Few Atomic Layers of Hexagonal Boron Nitride on Melted Copper. Scientific Reports 2015, 5, 7743

32. Wen, Y.; Shang, X. Z.; Dong, J.; Xu, K.; He, J.; Jiang, C., Ultraclean and 
large-area monolayer hexagonal boron nitride on $\mathrm{Cu}$ foil using chemical vapor deposition. Nanotechnology 2015, 26, 275601.

33. Nai, C. T.; Lu, J.; Zhang, K.; Loh, K. P., Studying Edge Defects of Hexagonal Boron Nitride Using High-Resolution Electron Energy Loss Spectroscopy. J Phys Chem Lett 2015, 6, 4189-4193.

34. Kiraly, B.; Iski, E. V.; Mannix, A. J.; Fisher, B. L.; Hersam, M. C.; Guisinger, N. P., Solid-source growth and atomic-scale characterization of graphene on $\operatorname{Ag}(111)$. Nat Commun 2013, 4, 2804.

35. Warner, J. H.; Rummeli, M. H.; Bachmatiuk, A.; Buchner, B., Atomic Resolution Imaging and Topography of Boron Nitride Sheets Produced by Chemical Exfoliation. Acs Nano 2010, 4, 1299-1304.

36. Bianchini, F.; Patera, L. L.; Peressi, M.; Africh, C.; Comelli, G., Atomic Scale Identification of Coexisting Graphene Structures on Ni(111). J Phys Chem Lett 2014, 5, 467-473.

37. Tang, S. J.; Wang, H. M.; Zhang, Y.; Li, A.; Xie, H.; Liu, X. Y.; Liu, L. Q.; Li, T. X.; Huang, F. Q.; Xie, X. M.; Jiang, M. H., Precisely aligned graphene grown on hexagonal boron nitride by catalyst free chemical vapor deposition. Scientific Reports 2013, 3, 2666

38. Lin, W. H.; Chen, T. H.; Chang, J. K.; Taur, J. I.; Lo, Y. Y.; Lee, W. L.; Chang, C. S.; Su, W. B.; Wu, C. I., A Direct and Polymer-Free Method for Transferring Graphene Grown by Chemical Vapor Deposition to Any Substrate. Acs Nano 2014, 8, 1784-1791.

39. Kidambi, P. R.; Blume, R.; Kling, J.; Wagner, J. B.; Baehtz, C.; Weatherup, R. S.; Schloegl, R.; Bayer, B. C.; Hofmann, S., In Situ Observations during Chemical Vapor Deposition of Hexagonal Boron Nitride on Polycrystalline Copper. Chem Mater 2014, 26, 6380-6392.

40. Jang, S. K.; Youn, J.; Song, Y. J.; Lee, S., Synthesis and Characterization of Hexagonal Boron Nitride as a Gate Dielectric. Scientific Reports 2016, 6, 30449.

41. Lee, G. H.; Yu, Y. J.; Lee, C.; Dean, C.; Shepard, K. L.; Kim, P.; Hone, J., Electron tunneling through atomically flat and ultrathin hexagonal boron nitride. Appl Phys Lett 2011, 99, 243114. 


\title{
Supporting information
}

\begin{abstract}
AFM images and photographs of bi-layer h-BN to 30-layer h-BN on $\mathrm{SiO}_{2} / \mathrm{Si}$ substrate and the height profile, infrared phonon of h-BN with the film thickness ranging from 30 layers to 1 layer, X-ray photoelectron spectra - $\mathrm{Cu} 2 \mathrm{p}$ spectrum of $1 \sim 30$ layers h-BN films, irreversible dielectric breakdown measurements to determine the hard-breakdown voltage, film thickness with respect to the Borazine partial pressure, growth mechanism of the h-BN synthesis by APCVD and LPCVD, SEM images showing triangular ad-layer with different domain size, cross - section TEM image of 5-layer h-BN, and AFM image of 5-layer h-BN with $0.69 \mathrm{~nm}$ root mean square roughness.
\end{abstract}


TOC.

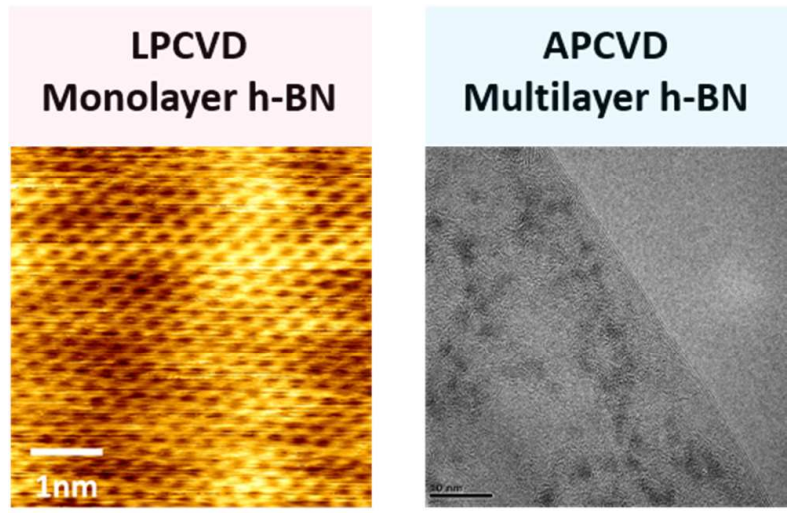

